\title{
Ciencias sociales en la educación infantil en entornos digitales: experiencias del profesorado
}

Social Sciences in early childhood education in digital environments: teachers' experiences

\author{
Miguel Angel Jara* \\ María Esther Muñoz ${ }^{* *}$ \\ Agustina Tirachini ${ }^{* * *}$
}

\section{RESUMEN}

Trabajar con instituciones infantiles diversifica la mirada y pone el acento en los sujetos del aprendizaje, entendiendo que los niños y las niñas viven una experiencia cultural diferenciada. Hay nuevas maneras de sentir, escuchar y ver en las múltiples pantallas que los/as rodean y que las enseñanzas son construcciones profesorales que se enmarcan en finalidades que son parte de la cultura digital de este tiempo. La experiencia investigativa nos vuelve a mostrar que enseñar ciencias sociales desde la infancia potencia las posibilidades del pensamiento y de las prácticas sociales críticas.

Palabras claves: educación inicial; ciencias sociales; cultura digital; enseñanza; aprendizaje.

\begin{abstract}
The work at children's institutions diversifies our consciousness and emphasizes learning subjects, because children live a distinct cultural experience. There are new ways to feel, hear and see in the multiple screens that surround the children, and teaching strategies are constructions that are part of the digital culture of this time. The experience of this research points out that teach Social Sciences since childhood enhances the possibilities of thought and of to criticize social practices.
\end{abstract}

Keywords: Early childhood education; social sciences; digital culture; teaching; learning.

\footnotetext{
* Profesor en historia y Doctor en Didáctica de las Ciencias Sociales. Profesor Adjunto Regular en el área de didáctica de las Ciencias Sociales e Historia para los profesorados en nivel inicial e historia. Facultades de Ciencias de la Educación y Humanidades de la Universidad Nacional del Comahue (FACE/UNCo, FAHum/UNCo).mianjara@gmail.com

** Profesora en historia y Especialista en Didáctica de las Ciencias Sociales. Profesora Auxiliar Regular en el área de didáctica de las Ciencias Sociales para los profesorados en nivel inicial y primario. Facultad de Ciencias de la Educación de la Universidad Nacional del Comahue (FACE/UNCo). munozmariaesther@gmail.com

*** Psicóloga. Profesora de evaluación psicológica en la carrera de psicología en la Facultad de Ciencias de la Educación de la Universidad Nacional del Comahue (FACE/UNCo). agustinatirachini@yahoo.com.ar
} 
La enseñanza de las ciencias sociales en la educación inicial de niñas y niños hasta el quinto año de edad se ha incorporado, masivamente, al curriculum de la educación argentina a principios de la década de 1980, abierto el proceso de democratización de la sociedad y sus instituciones. En la región del alto valle, provincias de Río Negro y Neuquén fueron procesos participativos de construcción curricular que, desde entonces a esta época, han sido modificados en varias oportunidades y en contextos de aplicación de política educativas de corte neoliberal.

Si bien la educación de niñas y niños en nuestro país ha sido una preocupación de larga data, podríamos asegurar que la misma no se organizó en torno a áreas del conocimiento escolar. Las disciplinas historia, geografía o educación ciudadana, que son las que con frecuencia se enseñan en el área de las ciencias sociales, no se incorporan sino hasta la década de 1960, del pasado siglo, en algunas jurisdicciones del país y masivamente con la reforma educativa de 1990. Es así como las ciencias sociales comienzan a tener un espacio reconocido en la estructura curricular, por lo tanto, se comienza a pensar qué contenidos de las ciencias sociales son apropiados para trabajarlos con las infancias de 4 y 5 años primero y luego desde los 45 días de edad. Un camino fundante en esta construcción fue recuperar experiencias del profesorado en ejercicio, cuestión que implicó una masiva participación y un claro corrimiento de la lógica de que el currículo y su construcción era solo cuestión de técnicos expertos, ahora éstos últimos acompañaban las demandas e intereses de los/as docentes.

La función educativa y social reconocida y atribuida al nivel, las perspectivas críticas sobre la educación, la sociedad, el estado y los sujetos fueron puntos de partida para la discusión, reflexión y elaboración de los diseños curriculares. Para ambos casos - las provincias de Río Negro y de Neuquén - que llevaron a cabo la construcción de los nuevos diseños, casi paralelamente, las ciencias sociales en el nivel ocupan un lugar privilegiado, en tanto subyace la idea de que todo contenido para el nivel deviene básicamente de este campo y se lo reconoce como un agente socializador importante. Para la primera jurisdicción se los aborda como campo de la realidad natural y social y para Neuquén como núcleos problemáticos que se definen por zonas.

En este marco general, en nuestra investigación, nos hemos propuesto conocer y comprender los múltiples y diversos contextos en los que se desarrollan las prácticas de la enseñanza y, fundamentalmente, indagar ¿cómo?, 
¿cuándo? y ¿por qué? el profesorado incorpora la cultura digital en la enseñanza y el aprendizaje las ciencias sociales en las salas de educación infantil.

\section{LAS CIENCIAS SOCIALES EN EL NIVEL INICIAL. RELATOS DE MAESTRAS QUE ENSEÑAN CIENCIAS SOCIALES}

Son varios los aspectos y las dimensiones que hemos considerado en el proceso y desarrollo de la investigación. A modo de presentar los semblantes de estas experiencias recuperamos las entrevistas realizadas ${ }^{1}$ a maestras que desarrollan su práctica en dos jardines de infantes de la provincia de Río Negro. Una corresponde al Jardín $\mathrm{n}^{\circ} 32$ de la ciudad de Bariloche y la otra al jardín $n^{\circ} 49$ de la ciudad de Cipolletti. ${ }^{2}$ Ambos casos nos dan cuenta de experiencias particulares, en el marco de realidades, institucionales, materiales y socioculturales concretas.

Reconocemos como valiosa la predisposición y apertura de las maestras para compartir con el equipo de investigación sus espacios de experiencias y de trabajo. De igual modo la de los directivos que facilitaron el trabajo con las docentes, para realizar las entrevistas, y nos ofrecieron los espacios físicos para que pudiéramos desarrollar nuestro trabajo. Las entrevistas se realizaron en el marco de las horas especiales ${ }^{3}$ por lo que no hubo inconvenientes para organizarlas según los tiempos disponibles.

Las maestras entrevistadas reconocen que esta actividad de trabajo e intercambio con el equipo y en el marco de un proyecto de investigación es favorable para su práctica y consideran que esto implica beneficios para la institución y los niños y las niñas. Lo que evidencia responsabilidad y compromiso con la tarea de enseñar en el marco de la cultura digital. Se reconoce necesaria la relación de la educación con las tecnologías y su integración como oportunidades de apropiación pedagógica, construida desde la escuela.

Del relato de las docentes - aunque sus prácticas se desarrollan en realidades escolares diferentes - se desprenden apreciaciones compartidas en torno a la incorporación e impacto de la cultura digital en la enseñanza y aprendizaje del conocimiento del medio social en el nivel.

Reconocen la influencia de la tecnología en la vida de los/as niños/as. Expresan que las instituciones educativas "funcionan" paralelamente al desarrollo 
de los medios tecnológicos. Los/as niños/as juegan y personifican a los/as protagonistas de los dibujos animados, también representados en videojuegos.

Los contenidos multimediales y su influencia en la cotidianeidad infantil atraviesan la tarea educativa. Las maestras sostienen que, inmersos en un mundo de expansión tecnológica, es necesario que los niños/as aprendan, en edades tempranas, a interactuar con el medio digital desde "otros lugares", que no sean solamente el de la "recreación o juego libre" y que para ello es importante establecer finalidades claras con relación a los contenidos que se quieren trabajar. Es decir "hacer que los/as niños/as jugando aprendan".

Entendidas como formas culturales las tecnologías modifican las experiencias cotidianas de los niños/as y las maneras en que conciben al mundo y se posicionan frente a él (De Angelis, 2010, p.106). En este sentido, las maestras entrevistadas, cuando manifiestan su uso en las propuestas educativas y destacan su valor didáctico, se ocupan de ello. Consideran importante el uso de los recursos tecnológicos como estrategias didácticas para el aprendizaje de los/as niños/as. La educación en el nivel, sostienen, debe favorecer en los/as niños/as la posibilidad de combinar imágenes, sonidos y movimiento en un mismo espacio virtual, que los invite a explorar, jugar y crear. Enfatizan en el desafío de acompañar potenciando esos sentidos y fortaleciendo las capacidades reflexivas y críticas, sobre los temas sociales que introducen en sus propuestas de enseñanza.

Para el caso del jardín de infantes de Bariloche y, ante la ausencia de soportes tecnológicos, promueven la presentación de un proyecto educativo en un evento gestionado por una empresa privada en el año 2010. ${ }^{4}$ De esta manera la institución, con el dinero que obtiene por la presentación del proyecto, incorpora una netbook, un retroproyector y una cámara de foto. Materiales tecnológicos que se utilizan para proyectar documentales, videos y crear escenarios para jugar en las salas con los niños/as. En los actos de fin de año para proyectar diferentes escenografías, videos con fotos que muestran a las familias las distintas actividades que los niños/as realizaron; en los actos escolares conmemorativos se utilizan para realizar videos donde los niños dramatizan pequeñas obras relacionadas con las efemérides.

Las docentes manifiestan que la falta de recursos condiciona el trabajo con las tecnologías y, por ende, su incorporación en las situaciones de enseñanza. El escaso involucramiento de los/as chicos/as con las tecnologías influye en 
el proceso de construcción del conocimiento escolar, si se aborda desde la perspectiva de la cultura digital. Los niños/as participan sólo en algunas ocasiones tomando fotos. No utilizan la netbook porque sólo hay una. Las docentes facilitan sus máquinas personales para que los niños/as puedan acceder o interactuar con algunos juegos-trabajo. También se utiliza la computadora de escritorio, que denominan como "vieja y obsoleta", en la que se ha incorporado juegos de matemática, rompecabezas, etc.

Ante esto se complica elaborar proyectos institucionales o planificar la enseñanza en función del uso de tecnologías. Sin embargo, cada docente las incorpora en la enseñanza a partir de inquietudes, preocupaciones y motivaciones personales. Reconocen las potencialidades y riqueza de incorporarlos en la enseñanza y el aprendizaje en las salas pero destacan que las tecnologías son una herramienta educativa donde las intencionalidades y finalidades pedagógicas no deben estar ausentes.

En sus expresiones enfatizan su desacuerdo con el uso de la tecnología con el objetivo solo de jugar. Si bien es un material que genera curiosidad y que muchos chicos acceden a las computadoras en sus hogares, el jardín debe posibilitar, presentar y brindar otras formas de interacción: "Desde conocer y aprender el para qué de su utilización que conlleve reforzar los contenidos a enseñar”.

En la enseñanza de contenidos sociales opinan que puede potenciar y enriquecer las miradas de los niños/as sobre el mundo social a partir de la observación y experimentación. Los chicos pueden percibir nociones temporales y espaciales con menores dificultades. El tema de la alteridad se puede trabajar con el uso de recursos diversos, concretos, vivenciados. Favorece la posibilidad de estrategias de comparación con viajes interactivos en espacios y tiempos distintos. Consideran que la incorporación de las tecnologías facilita y enriquece el desarrollo de capacidades y miradas diversas; facilitan la tarea educativa y mejoran la calidad de la educación.

El predominio de la imagen, la velocidad, los mensajes y discursos fugaces cobra presencia en el mundo infantil y el desafío, para las docentes, es enseñar generando y promoviendo aprendizajes que modifiquen la concepción de lo tecnológico como entretenimiento que los niños puedan haber incorporado. ${ }^{5}$ 
Sostienen la importancia del lugar docente que "no deja de ser visible... está siempre presente, más aún en el nivel inicial donde los niños/as requieren de un comprometido acompañamiento". Consideran que los actuales procesos de digitalización de la cultura implican también cambios en el diálogo y la comunicación social y como docentes deben guiar, coordinar y orientar el involucramiento e interacción en edades tempranas. El uso de la tecnología y su presencia en educación no resuelve el acto educativo: "Sí enriquece, facilita como recursos, sí agiliza o moviliza... no reemplaza al docente”.

La complejidad de los contextos en el que se desarrollan las prácticas de enseñanza pone en relieve diversidad de realidades donde los modos particulares son objeto de reflexión didáctica.

Del relato de las maestras, que si bien manifiestan no planificar propuestas a partir del uso de las tecnologías por las dificultades en equipamiento y materiales didácticos, se evidencia su implicación en el trabajo didáctico con los niños/as en situaciones aisladas y esporádicas.

El jardín $n^{\circ} 49$ de Cipolletti con el ingreso al programa provincial, es una de las primeras instituciones de educación infantil, de tres en la ciudad, que fue equipada con un aula digital móvil integrada por 14 netbook para los/as niños/as, una para uso docente, un cañón reproductor y diversas aplicaciones electrónicas; además de las conexiones técnicas informáticas.

La presencia de infraestructura, si bien no es un factor determinante en la utilización de tecnologías, su ausencia o limitaciones tiene cierta correspondencia en el uso institucional. En este sentido la institución no dispone de un espacio para los soportes tecnológicos recibidos. En varias ocasiones solicitaron a las autoridades ministeriales un gabinete móvil para guardar las computadoras y ser trasladadas de forma segura y fácil a las salas; así como el pedido de otras computadoras porque son 19 los niños/as por sala. Sin respuesta y frente a la urgencia de la situación se utilizó como espacio físico el único armario que se encuentra en una de las salas-aulas de la institución. Para trabajar en las respectivas salas, las docentes con los niños deben retirarlas del armario interrumpiendo, inevitablemente, el desarrollo de las actividades en la sala-aula donde se encuentran.

Con la disponibilidad del Aula Digital, los docentes diseñaron un "Proyecto de Computación" donde el propósito fundamental consistía en "promover 
actividades y utilizar programas que estimulen los sentidos, la creatividad, el pensamiento y alfabetización infantil”.

De esta forma se propusieron mediante el uso de los recursos tecnológicos, como la computadora, potenciar y desarrollar estrategias de trabajo donde los niños/as se involucren e interactúen en un ámbito de aprendizaje colectivo.

La propuesta de trabajo fue posible sin dificultades el primer año del ingreso al programa. Se realizaron muestras abiertas a la comunidad educativa en la que lo/as niños/as de las salas de 5 años comentaron y compartieron, junto a las maestras, el trabajo realizado con los recursos tecnológicos durante el año. Las maestras de cada una de las salas de ambos turnos - mañana y tarde - abordaron diferentes temáticas como los derechos del niño, la cultura mapuche, el ciclo del agua, entre otras.

Las docentes destacan que las conexiones en red y el funcionamiento de los dispositivos posibilitaron el desarrollo e implementación de las propuestas educativas en el marco del proyecto institucional.

Lamentablemente este proyecto no tuvo continuidad en el tiempo. En los sucesivos años, los problemas de mantenimientos de las computadoras, de conexión, de funcionamiento técnico son obstáculos frecuentes. Si bien cuentan con el asesoramiento y acompañamiento de referentes pedagógicos tics, que comparten con las otras instituciones del nivel y con el nivel primario, y acuden cuando se los solicita, sostienen que el mantenimiento no es regular. Es usual que las netbook se "tilden", que se presenten problemas de configuración y dificultades para conectarse en red. Resaltan enfáticamente, en sus relatos, que los problemas no refieren "al mal uso" ni a la incorrecta manipulación de los niños/as. Contrario a ello son cuidadosos y responsables. Los artefactos como tal se encuentran en buenas condiciones y no han sufrido ningún tipo de daño material. Los niños/as nunca están solos cuando trabajan en la sala con las computadoras y esperan atentamente las diferentes indicaciones y orientación de las maestras.

Las experiencias cotidianas implican procesos de marchas y contramarchas; organización reorganización y nuevos desafíos. Durante el momento de trabajo del equipo de investigación con la institución se había acordado entre las docentes, para ese año, un día determinado de la semana para la utilización y trabajo, en las diferentes salas, con las netbook y el proyector. 
Según los relatos profesorales muchos chicos/as no tienen acceso a una computadora en sus hogares y que los problemas de conexión a internet son una constante en el barrio. Sin embargo, los/as chicos/as acceden a la tecnología a partir del uso de los celulares de sus madres que con el acelerado avance tecnológico "son como una mini-computadora".

Estos problemas, obstáculos y dificultades con los recursos tecnológicos, algunos de ellos imposibilitados de utilizar, impactan en el desarrollo de las propuestas planificadas y en ocasiones imposibilita su concreción en función de los propósitos pedagógicos.

Las entrevistas en profundidad a las docentes nos muestran datos que se relacionan con las dificultades, pero no significan problemas o resistencia a las nuevas innovaciones que responden a cambios socioculturales. Se observa el compromiso y trabajo de la institución y de las docentes en sus propuestas de enseñanza y en pos de promover el contacto de los/as niños/as con el uso de las tecnologías, reconociendo su importancia en contextos de inclusión social y cultural. En sus relatos pareciera que lo tecnológico se concibe como un recurso didáctico importante para el aprendizaje en la primera infancia. Se promueven experiencias educativas con el objeto de potenciar espacios valorando la apertura, apropiación e interés por parte de los/as niños/as.

Experiencias cotidianas que en el espacio escolar tienen como finalidad abrir nuevos horizontes en los que los/as niños/as sean activos partícipes en estos contextos epocales del que son protagonistas (De Angelis, 2010).

Las prácticas educativas, como prácticas sociales, se sumergen en procesos de construcción permanente. Si el entramado institucional en los centros educativos reviste de gran complejidad en los procesos de innovaciones aceleradas interpela y plantea nuevos desafíos, preocupaciones y complejidades.

\section{UNA EXPERIENCIA CONCRETA DE ENSEÑAR CIENCIAS SOCIALES CON CULTURA DIGITAL}

La experiencia concreta que queremos compartir con el/la lector/a fue realizada con una maestra del Jardín $\mathrm{N}^{\circ} 49$ de la ciudad de Cipolletti. La sala de 5 años, turno tarde, está conformada por 17 niños/as. En la sala asistía una niña integrada, acompañada por una maestra de apoyo a la inclusión (MAI) ${ }^{6}$ El número de niños/as, en esta sala, es menor a la matrícula de las otras salas 
de la institución, de manera que se pueda garantizar la trayectoria educativa integral de la niña. La docente trabaja hace tres años en la institución y su ingreso coincidió con la puesta en marcha del programa digital en el año 2012. Fue una de las docentes que participó en la elaboración e implementación del proyecto institucional de computación.

Según expresiones de la docente, actualmente, el equipamiento recibido se utiliza "algunas veces como recurso didáctico en relación a la unidad que estamos trabajando, para poder ver algo en el proyector. Y otras veces lo usamos como un recurso en sí. Para usar los juegos que tenemos con contenidos matemáticos, tenemos bastantes juegos".

Se intenta de esa forma, dar continuidad durante todo el ciclo lectivo al proyecto educativo institucional. En la sala de cuatro, ${ }^{7}$ los niños comienzan a utilizar las computadoras a mitad de año por cuestiones de adaptación a la institución, al grupo y la construcción de vínculos con las docentes. El trabajo consta de dos momentos diferenciados. El primer momento es de exploración, de conocimiento, involucramiento de los/as niño/as con la herramienta/máquina. Es decir de contacto con las diferentes funciones, programas, ubicación de aplicaciones, etc. El segundo momento implica complejizar, a partir del diseño de actividades didácticas con contenidos específicos.

Las salas de cinco comienzan a trabajar desde el principio del año porque la mayoría de los/as niño/as asistieron al jardín el año anterior por lo que ya tienen experiencia en el aprendizaje con tecnología. Una vez a la semana cada sala utiliza las netbooks. La maestra comenta que los/as niño/as reconocen el día y esperan el momento de trabajo, además de disfrutarlo mucho...

La experiencia a compartir se desarrolla en el marco de uno de los proyectos diseñado y planificado por las docentes que articula la temática "conocemos nuestras raíces". La secuencia corresponde al contenido vinculado con la música y los instrumentos musicales.

Las actividades didácticas son pensadas entre treinta y cuarenta minutos. Decisión que se fundamenta en la atención de los niño/as; las condiciones de las máquinas y las conexiones.

En este sentido la docente debe planificar considerando varios aspectos que no sólo refieren al sujeto que aprende; las posibilidades educativas; finalidades; material educativo sino también funcionamiento de los soportes tecnológicos. El día que corresponde su utilización, la docente en la hora en que los 
niños/as se encuentran en la clase de música verifica las condiciones de los artefactos. Además de cerciorarse, al inicio de la jornada, que las baterías estén cargadas.

El material educativo seleccionado por la docente son los juegos digitales. Con ello se intentaba fomentar la adquisición de habilidades y capacidades de exploración, observación, interacción grupal, curiosidad, libertad para experimentar y disfrutar. El desarrollo de habilidades cognitivas, comunicativas, identitarias y de autonomía orientaron la planificación de la propuesta. Además en la lectura de los registros se destacan cómo se generan actitudes relacionadas con el uso y cuidado de las máquinas; el respeto por los acuerdos previos y la colaboración entre pares.

El trabajo en el aula comienza con la organización para buscar las netbook en el armario que se encuentra en la sala verde de cuatro años. La docente es acompañada por grupos de cuatro niños/as -según la ubicación en las mesas-, para retirar una máquina y regresar a la sala, momento en el que sale otro grupo hasta completar la acción. Este momento es realizado con gran responsabilidad de parte de los/as niño/as. Regresan caminado despacio y en "orden" sujetando las máquinas con las dos manos; se sientan en sus respectivos bancos; no las abren/tocan o encienden y esperan que el resto de los compañeros regresen. Comentan entre sí el número de la máquina que se les asignó y simulan que se conectan en internet -siempre con las máquinas apagadas y cerradas-. Esperan pacientemente el comienzo del trabajo planificado para la clase.

Actitudes que en el proyecto institucional aparecen como propósitos favorables a fomentar en los niño/as. Además se visualiza el trabajo en el manejo y reconocimientos de los elementos de la computadora e interacción que correspondió a la programación del año anterior cuando asistieron a la sala de cuatro. Lo que implicó la experiencia de estar en contacto con la lógica de funcionamiento y modos particulares de interacción que impone la máquina a los/as niños/as usuarios. El carácter interactivo permite que los/as niños/as tengan respuestas inmediatas sobre las acciones que en ellas realizaron (De Angelis, 2010).

El trabajo se realiza con pequeños grupos debido a los problemas de conexión. Sólo cuatro netbook son conectadas a internet. Se propone juegos interactivos para experimentar y crear sonidos musicales con diferentes instrumentos; el trabajo de coordinación y reconocimiento de secuencias. El resto 
de la clase elige alguno de los juegos que se encuentran en las computadoras hasta esperar su turno. Estos juegos van incrementando su complejidad en tres niveles.

El escaso acceso al recurso tecnológico, en sus hogares, no es un impedimento en el aprendizaje de los/as niño/as. Escuchan atentamente la explicación y coordinación de la maestra que va expresando a la clase las acciones a seguir y el contenido curricular. Asimismo observan, interactúan con la docente, plantean hipótesis sobre las formas que consideran más exitosas para transcurrir o superar los obstáculos y desafíos que les presentan los juegos interactivos. Lo que significaba avanzar hacia los siguientes niveles de mayor complejidad en el juego.

La decisión de la aplicación de la metodología lúdica se corresponde con concepciones que subyacen en los decires y en el diseño de la propuesta didáctica de la maestra acerca de ponderar su potencialidad educativa y su relación con el contenido. En este caso el juego que asume una forma digital, con intencionalidades didácticas se desdibujan o entrelazan con preocupaciones y responsabilidades con la enseñanza en los actuales contextos sociales, institucionales y laborales.

En este sentido la docente ofrece a los/as niño/as el juego como experiencia de aprendizaje y como modo de aproximación al contenido. La situación lúdica se considera de importancia, potencialmente pertinente para que el aprendizaje suceda (Rodríguez Sáenz, 2010). Aprendizaje vinculado a trabajar un contenido específico del área, pero también a la incorporación de la tecnología que no responden sólo a consideraciones manifiestan como recurso. Se asume fundamental importancia al soporte mismo y sus implicancias para el trabajo didáctico con los/as niño/as.

La docente orienta en las diferentes acciones referidas al involucramiento y contacto en la situación de enseñanza con lo digital. Estimula y responde a las inquietudes de los estudiantes. Cada desafío implica operaciones mentales de parte de los/as niños/as y son acompañados por la maestra, quien además promueve el trabajo colaborativo entre pares. El uso de las tecnologías cobra así sentido porque orienta construcciones cognitivas en torno a las situaciones sociales. Además desarrollar habilidades vinculadas a compartir, intercambiar, interactuar, argumentar y fundamentar opciones en los juegos con tecnología (De Angelis, 2010, p.124). 


\section{REFERENCIAS}

ALBA, Alicia de. Curriculum: crisis, mito y perspectivas. Buenos Aires: Miño y Dávila, 1998.

DE ANGELIS, Susan. E. El juego como apertura a nuevos mundos. In: SARLÉ, Patricia (Coord.) Lo importante es jugar: cómo entra en juego en la escuela. Rosario: $\mathrm{H}$. Sapiens, 2010. p.105-124.

DUSSEL, Inés. Aprender y enseñar en la cultura digital. Buenos Aires: Santillana, 2010.

RODRÍGUEZ SÁENZ, Inés. El juego como contenido. In: SARLÉ, Patricia (Coord.) Lo importante es jugar: cómo entra en juego en la escuela. Rosario: H. Sapiens, 2010. p.75-88.

\section{NOTAS}

${ }^{1}$ En el primer y segundo artículo de este dossier, hemos desarrollado los procesos metodológicos que nos han permitido situar y comprender la diversidad de las prácticas en las aulas del nivel en instituciones educativas públicas.

${ }^{2}$ Jardines que se ubican en la Zona Andina y Alto Valle de la provincia de Río Negro.

${ }^{3}$ Son las horas en las que él o la maestra a cargo de la sala tiene un tiempo liberado, en tanto el grupo de niños/as queda a cargo de profesores/as de Educación Física, Música, Teatro u otras asignaturas.

${ }^{4}$ La institución participa y justifica en el proyecto la necesidad de contar con una netbook, un retroproyector y una cámara de foto para recabar información, para poder bajar láminas y trabajar con los chicos en las diferentes salas. El concurso impulsado por una empresa de telefonía otorgaba dinero a los proyectos seleccionados.

${ }^{5}$ De la lectura de los decires docentes se visualiza gran preocupación del lugar que ocupan las computadoras en los hogares de los niños. Plantean: "no se trata de reemplazar el espacio, que en otras épocas, ocupaba el televisor hoy lo ocupe la computadora. No significa la computadora como recurso para jugar sino para abordar contenidos, para aprender". De allí que según sus relatos se trata de utilizar las tecnologías en relación a la enseñanza, al objeto a enseñar.

${ }^{6}$ En la provincia de Río Negro se encuentra en vigencia el Proyecto de Integración de alumnos/as con discapacidad al sistema educativo común en los distintos niveles y modalidades (Res. nº 996/06del Consejo Provincial de Educación). En el año 2011 según Res. no 03438/11 se crea la figura de un maestro integrador en discapacidad mental, auditiva, visual y motora denominado "Maestro de Apoyo a la inclusión" (MAI). Y se establece que los establecimientos educativos de Nivel Inicial y Primario pueden requerir de apoyos in- 
dividualizados intensivos y/o permanentes de un/a MAI. con sede en la escuela donde se incluye el/a alumno/a.

${ }^{7}$ Se identifican las saladas de acuerdo a las edades de las/os niñas/os. Actualmente el nivel inicial (maternal y jardín) hay salas de 45 días a 5 años de edad.

Artigo recebido em 8 de abril de 2016. Aprovado em 30 de maio de 2016. 